\title{
The effect of hydrogen nanobubbles on the morphology of gold-gelatin bionanocomposite films and their optical properties
}

\author{
M Alsawafta ${ }^{1}$, S Badilescu ${ }^{2}$, Vo-Van Truong ${ }^{1}$ and M Packirisamy ${ }^{2}$ \\ ${ }^{1}$ Physics Department, Concordia University, 1455 de Maisonneuve Boulevard West, Montréal, QC, \\ H3G 1M8, Canada \\ ${ }^{2}$ Mechanical and Industrial Engineering Department, Concordia University, 1455 de Maisonneuve \\ Boulevard West, Montréal, QC, H3G 1M8, Canada
}

E-mail: sbadiles@ece.concordia.ca

Received 12 October 2011, in final form 24 November 2011

Published 17 January 2012

Online at stacks.iop.org/Nano/23/065305

\begin{abstract}
Gold-gelatin bionanocomposite films are prepared by the reduction of gold ions by sodium borohydride in an aqueous solution. It is shown that both the solution and the films on glass substrates contain entrapped hydrogen micro- and nanobubbles with diameters in the range of $200 \mathrm{~nm}-3 \mu \mathrm{m}$. The optical properties of gold nanoparticles in the presence of gelatin and hydrogen nanobubbles are measured and simulated by using the discrete dipole approximation method. The composite films having micro- and nanobubble inclusions have been found to be very stable. The calculated localized surface plasmon resonance band is found in agreement with the experimental band position only when the presence of hydrogen bubbles around the gold nanoparticles is taken into account. The different morphological features engendered by the presence of the bubbles in the film (gelatin receptacles for the nanoparticles, gelatin hemispheres raised by the bubbles under the surface, cavities on the surface of the film, etc) are described in detail and considered for potential applications. This work is highly relevant to the new and exciting topic of nanobubbles on surfaces and interfaces.
\end{abstract}

(Some figures may appear in colour only in the online journal)

\section{Introduction}

Metal-polymer nanocomposite (or nanometal-polymer composite) films are hybrid materials with inorganic nanoparticles immobilized and integrated into a polymer matrix. Upon the combination of the properties of the two fundamentally different materials, novel functions can be generated, leading to a wide range of interesting applications. The polymer host provides processibility and, in many cases, transparency and, by immobilizing the metal nanoparticles, protects them against aggregation or segregation. On the other hand, metal nanoparticles such as gold ( $\mathrm{Au})$ or silver $(\mathrm{Ag})$ significantly contribute with their distinctive optical properties. In order to emphasize their major contribution to the overall optical properties of the hybrid material, they are generally called 'optically effective additives' [1]. Nanocomposites may show optical nonlinearities and/or ultralow or ultrahigh refractive indices and are suitable for applications such as color filters, optical sensors, data storage, waveguides, etc [2-4].

More recently, a new class of composites, called bionanocomposites (BNC), has emerged. In this case, natural polymers such as chitosan, gelatin and polysaccharides, such as starch, are used to stabilize the metal nanoparticles against aggregation [5]. Among the advantageous properties of natural polymers are biocompatibility, biodegradability and non-toxicity for the environment. They have been proved to be suitable for the 'green' synthesis of gold and silver 
nanoparticles [6]. In some cases, the natural polymers can be used as both a stabilizer and thermal reducing agents [7, 8].

Gelatin is a soluble polypeptide, a denaturated product of the naturally occurring collagen. It is obtained by a controlled hydrolysis from the fibrous insoluble protein collagen and has been used for a long time in food processing, the pharmaceutical industry and medical applications. In the biologically active form, gelatin has a triple-stranded $\alpha$-helix structure. Below the isoelectric point $(p I=4.7)$, it contains both acidic and basic charged functional groups $\left(\mathrm{NH}_{2}\right.$, $-\mathrm{COOH},-\mathrm{SH})$ that may interact with the metal nanoparticles. Gelatin bionanocomposites of silver nanoparticles have also been prepared by using strong reducing agents such as sodium borohydride and the average diameter of nanoparticles was found to be around $4.5 \mathrm{~nm}$ [5].

In an attempt to prepare gold-gelatin BCNs with well-dispersed gold nanoparticles, we have used sodium borohydride $\left(\mathrm{NaBH}_{4}\right)$ as a strong reducing agent. The reaction takes place with the formation of Au nanoparticles and a large amount of gaseous hydrogen as shown by the equation below:

$$
\begin{aligned}
& \mathrm{Au}^{3+} / \text { gelatin }+\mathrm{BH}_{4}^{-}+3 \mathrm{H}_{2} \mathrm{O} \rightarrow \mathrm{Au}^{0} / \text { gelatin } \\
& \quad+\mathrm{B}(\mathrm{OH})_{3}+3.5 \mathrm{H}_{2} .
\end{aligned}
$$

We have observed that, under the conditions of our experiment, the hydrogen bubbles are trapped in the gold-gelatin solution and in films deposited from the solution on a glass substrate. The size of the hydrogen bubbles varies between $200 \mathrm{~nm}$ to approximately $2 \mu \mathrm{m}$ and the coexistence of the gas and gold-gelatin phases provokes the emergence of some amazing morphological features.

Surface nanobubbles are emerging as a new class of quite stable structures that may alter many aqueous interfaces and affect phenomena such as biocompatibility, adhesion between hydrophobic surfaces in water, etc. They are believed to be stabilized electrostatically when they are formed spontaneously on hydrophobic surfaces [9]. The most direct experimental evidence of the existence of nanobubbles at the liquid-hydrophobic surface comes from AFM images [10]. It has to be noted that, contrary to the predictions of thermodynamic calculations, the lifetime of nanobubbles is very long (hours to months), depending on surface roughness, $\mathrm{pH}$, concentration of electrolytes, temperature and pressure. Nanobubbles have been used as templates to form hollow $\mathrm{Au}$ nanoparticles [11] as well as nanoporous conductive polymer films [12] and core-shell nanoparticles [13]. It has been shown that, next to the tremendous scientific interest attracted by nanobubbles, they also have a great potential for applications, for example in nanofluidics, for ultrasound diagnostics, gaseous drug delivery, etc. For a comprehensive review on nanobubbles, see $[14,15]$.

In the present work, we are exploring the optical properties and morphological features of Au-gelatin nanocomposite films having hydrogen nano- and microbubble inclusions. This represents a sealed system, where nanobubbles are trapped by the drying of the film and, thus, cannot leave by diffusion. We attempt to develop structures where the gold nanoparticles are directly associated with nanobubbles and we investigate the stability of these systems, in solution as well as in films. In the case investigated here, the hydrogen micro- and nanobubbles are generated chemically, through the reduction of chloroauric acid by sodium borohydride, and trapped at the surface of the film by drying. Tapping mode AFM is utilized to investigate the morphology. Simulation of nanocomposite materials in the presence of nanobubbles by using the discrete dipole approximation (DDA) method are presented as well.

\section{Experimental details}

Gelatin from gold water fish skin was purchased from Sigma-Aldrich, sodium borohydride from Fluka and gold chloride from Alfa Aesar.

The gold-gelatin nanocomposite is synthesized as follows: typically $150 \mathrm{mg}$ of gelatin is dissolved in $50 \mathrm{ml}$ of DI water under stirring and heating to $50{ }^{\circ} \mathrm{C}$. After the dissolution, the gelatin solution is cooled down to room temperature. $50 \mathrm{mg}$ of $\mathrm{HAuCl}_{4} \cdot 3 \mathrm{H}_{2} \mathrm{O}$ is dissolved separately and added to the freshly prepared gelatin solution under stirring. Finally, a solution of sodium borohydride $(60 \mathrm{mg}$ in $5 \mathrm{ml}$ of water) is added drop by drop to the mixture, before bringing the final volume to $75 \mathrm{ml}$. The solution was then stirred for $15 \mathrm{~min}$ at room temperature and cast on a pre-cleaned glass substrate. Experiments have been performed by varying the molar ratio of sodium borohydride to chloroauric acid from 12.5 to 2.2 , respectively. The film was left for approximately one day to dry in the atmosphere.

UV-visible spectra were measured using a Perkin Elmer (Model Lambda 650) spectrophotometer and the AFM instrument Model NSCRIPTORTM DPN ${ }^{\circledR}$ System DS006 (Nano INK) was used for imaging the samples.

\section{Results and discussion}

\subsection{Optical properties of Au-gelatin nanocomposite solutions and films with trapped hydrogen nanobubbles}

After the sodium borohydride solution was added (in a large excess compared to the stoichiometric requirement of the reaction) to the dissolved gelatin, the color of the solution turned immediately to red because of the formation of gold nanoparticles. At the same time, a large number of bubbles of hydrogen, that results from the reaction, can be seen in the solution and above it. The reduction reaction was completed after approximately $15 \mathrm{~min}$. Figure 1 shows the reddish foam on the top of the solution and the inset shows the appearance of the film on a glass substrate. It can be seen that the film has trapped large bubbles, together with small ones which cannot be seen. The UV-visible spectrum of the freshly prepared gold-gelatin nanocomposite is dominated by the Au-localized surface plasmon resonance (LSPR) band as shown in figure 2.

Figure 2 shows that both the solution and the film are stable for months. The Au LSPR band is found at $510 \mathrm{~nm}$ in the solution and at $522 \mathrm{~nm}$ in the film. The position of the band corresponds to small nanoparticles with a diameter inferior to $10 \mathrm{~nm}$ [16-18]. However, the presence of bubbles surrounding the Au nanoparticles may influence significantly the position of the band, that is, the nanoparticles may be larger than the 


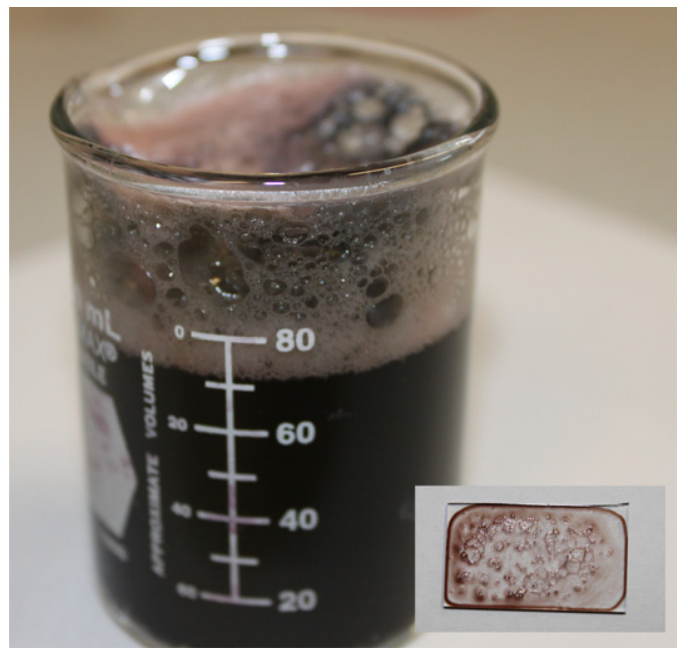

Figure 1. The aqueous solution of the $\mathrm{Au}$-gelatin nanocomposite with hydrogen bubbles on top. The inset shows the nanocomposite film on a glass substrate.

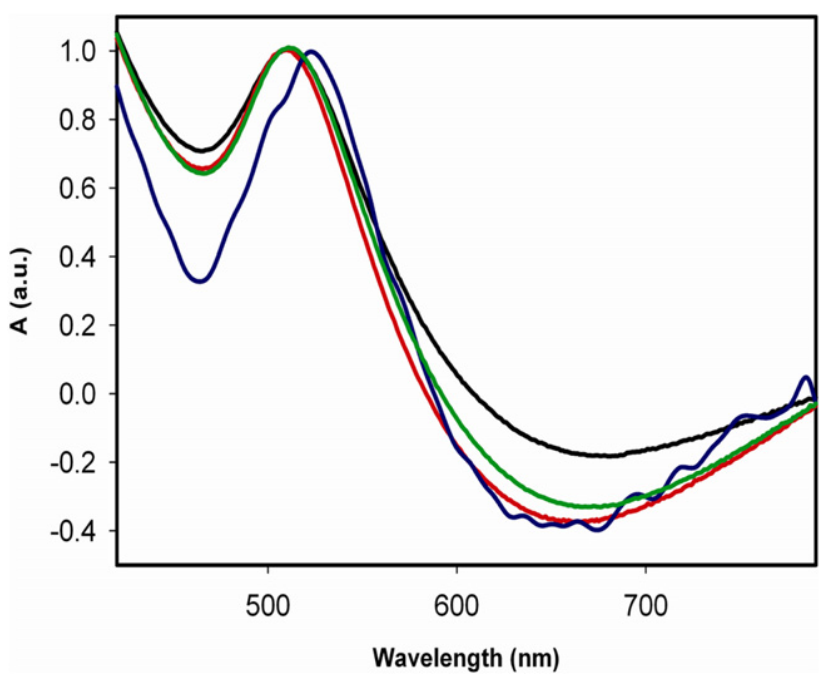

Figure 2. Au LSPR band of gold in the gold-gelatin nanocomposite, in the presence of hydrogen bubbles (red curve: solution, one month from the synthesis; green curve: solution, two months from the synthesis; blue curve: film on glass).

size predicted from spectral data in aqueous solutions. The high stability of the nanocomposite is accounted for by the stabilizing effect of the electron donor groups of the gelatin as well as, most probably, of the hydrogen bubbles [19]. However, at this stage, it is difficult to discriminate between the two effects.

\subsection{Morphological features of the Au-gelatin nanocomposite film with hydrogen bubble inclusions}

Au-gelatin films were cast from the solution on glass substrates and, after drying in the atmosphere, they were imaged by AFM in the tapping mode. The images show that neither the gold nanoparticles nor the bubbles are distributed uniformly in the dried film. The images below were selected

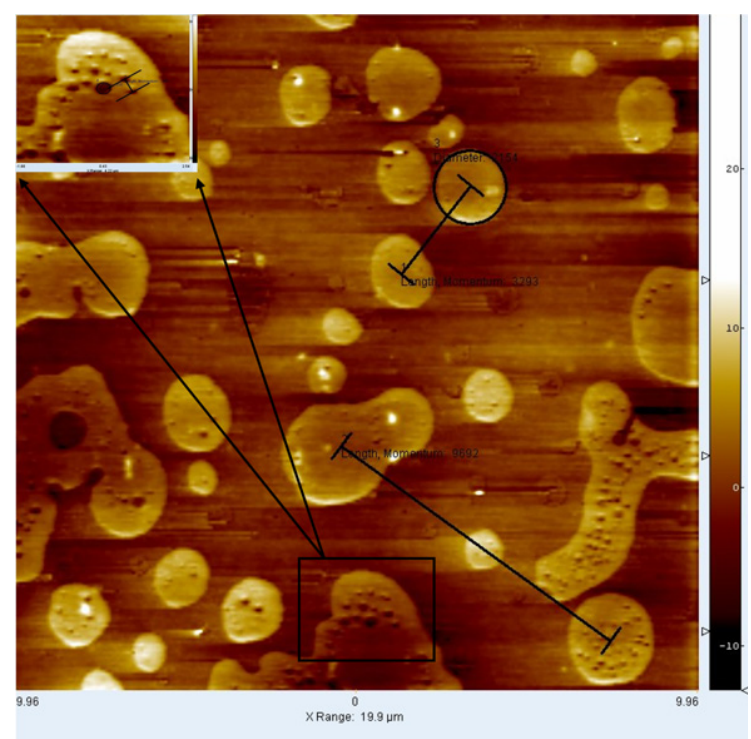

Figure 3. AFM image of Au-gelatin nanocomposite film showing the gelatin patches containing nanobubbles. The inset shows the bubbles trapped inside a gelatin patch.

with the aim of showing the most intriguing morphological features found in the films.

Figure 3 shows gelatin patches, mostly of circular shapes, with inclusions of bubbles and only a small number of gold nanoparticles. It can be seen that the gelatin patches are large (around 2-3 $\mu \mathrm{m}$ and the trapped bubbles are of the order of 300-400 $\mathrm{nm}$ and well separated.

The spherical structures and annular pores of catfish skin gelatin aggregates were studied by using AFM and discussed [20, 21]. Figure 4 shows another area $(20 \mu \mathrm{m} \times 20 \mu \mathrm{m})$ of the same film where, exclusively, gold nanoparticles can be found. Both 2D and 3D images show that the particles are fairly uniformly distributed on the surface of the film and their approximate sizes are 100-200 nm.

Figure 5 shows very clearly the presence of $\mathrm{Au}$ nanoparticles in the middle of the circular traces left behind by the bubbles that diffused from the film and exploded at the film-air interface. In this case, the gelatin surface is raised by the diffusion of bubbles and the trace becomes a kind of receptacle for nanoparticles. The figure shows that there are several such traces containing one nanoparticle each, and others, with 2-3 or more nanoparticles. We believe that the bubbles, in their way to the surface, draw the nanoparticles and carry them along. By controlling the diffusion of the bubbles, it becomes possible to carry and deposit nanoparticles at well-defined places, that is, to pattern the surface of the film. As can be seen in the figure, some of the traces are empty, meaning that the bubbles left the film without meeting nanoparticles. The size of the bubbles in this image are around $300 \mathrm{~nm}$. The figure shows that some parts of the film are raised by bubbles trapped under the surface of the film.

Figure 6 shows the bubbles of hydrogen close to the surface, inside the nanocomposite film. Only one $\mathrm{Au}$ nanoparticle can be seen, at the bottom, in the right corner 

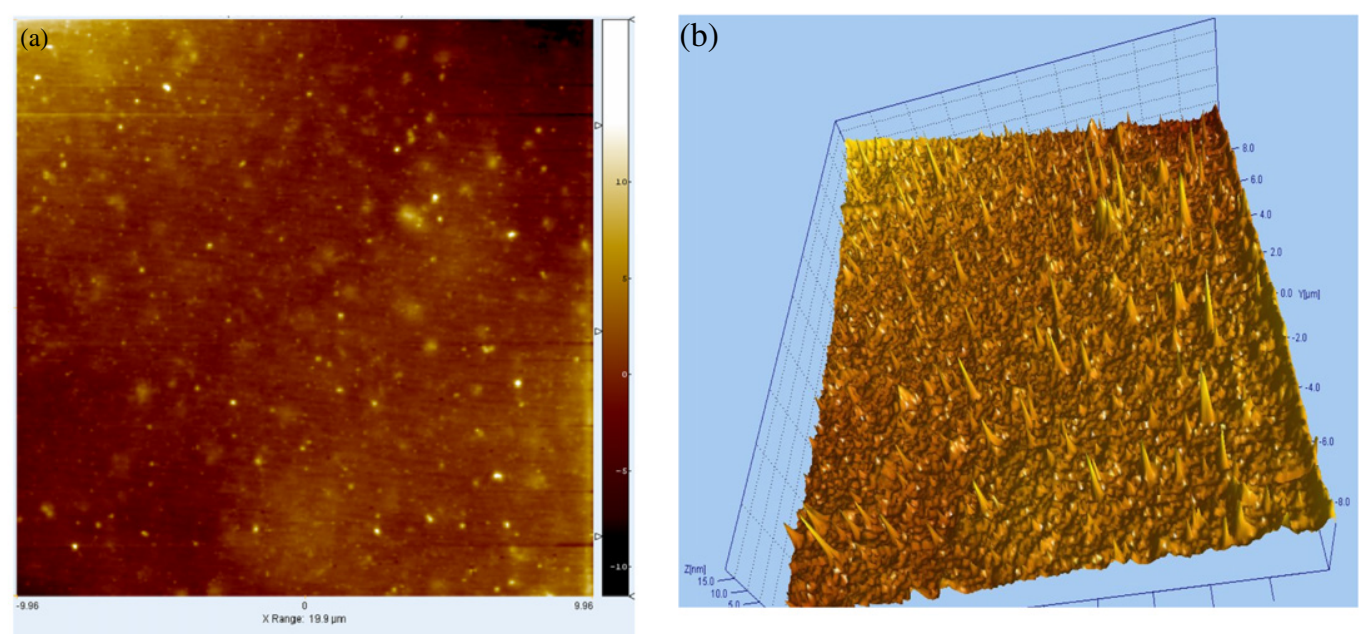

Figure 4. AFM images of Au-gelatin nanocomposite showing the distribution of gold nanoparticles in the film. Gold nanoparticles appear as white dots in the 2D image and sparks in the 3D image.
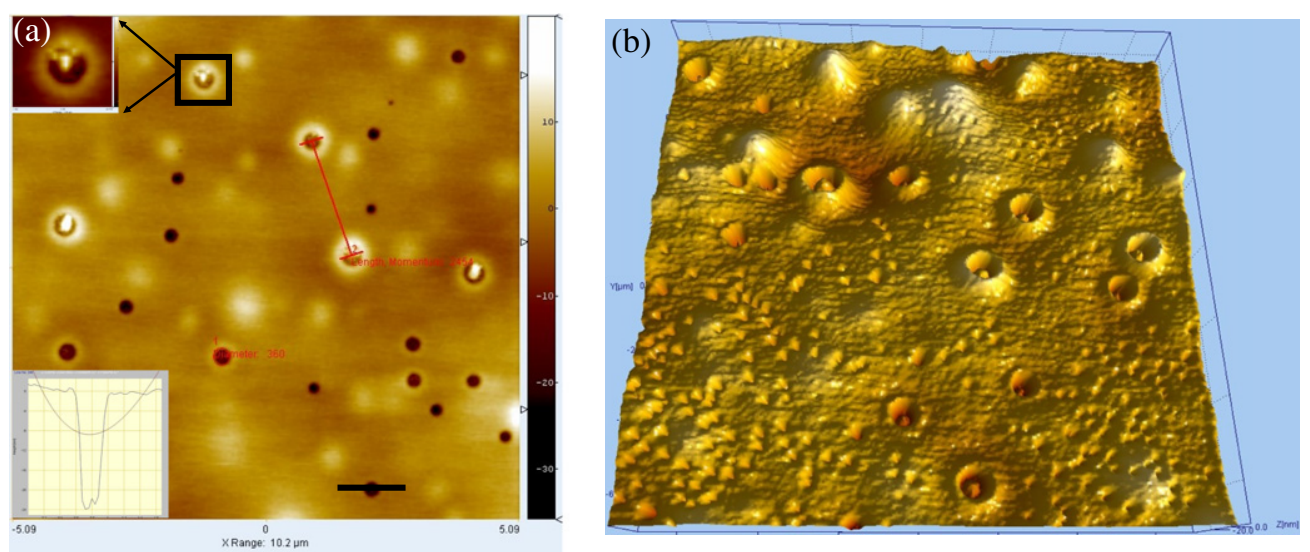

Figure 5. AFM image of the traces of exploded bubbles with trapped gold nanoparticles in the middle. The inset shows the enlarged image of one of the traces that have trapped gold nanoparticles. The distance between two neighboring bubbles is around 2-3 $\mu \mathrm{m}$ and the depth of the lowest bubble (indicated in the figure) is around $13 \mathrm{~nm}$.
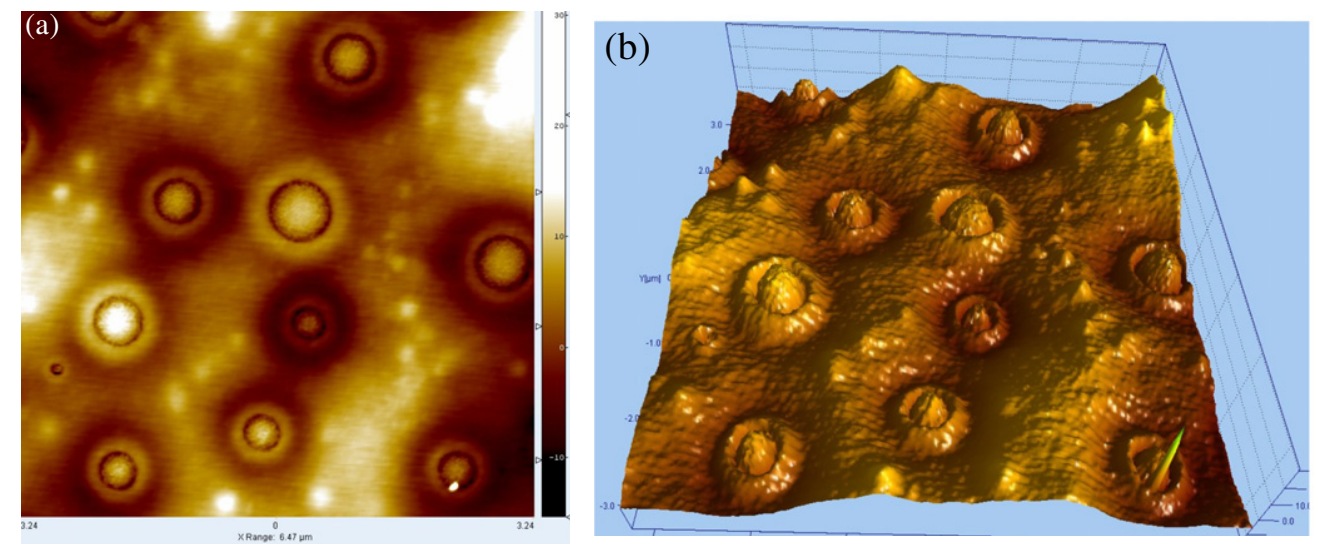

Figure 6. AFM images of the bubbles in the gelatin matrix, under the surface of the film.

of the image. All the other features show convex hemispheres that correspond to the raised gelatin film surface. In this case, it seems obvious that all the bubbles are still stable, under the surface of the dried film. They were, probably, deeper in the solution and had not enough time to reach the surface and explode before the film dried. 

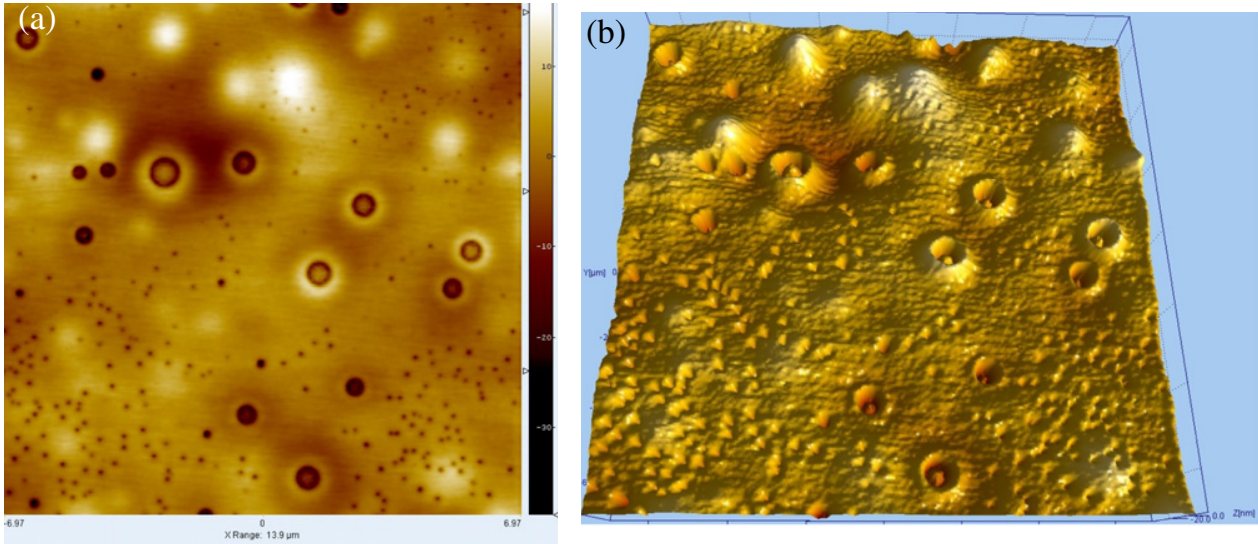

Figure 7. AFM images of nanobubbles deep inside the film (dark circles in the $2 \mathrm{D}$ image) and of those close to, or on, the surface, respectively. Cavities on the surface of the gelatin film are left behind by the bubbles exploding during the drying process.
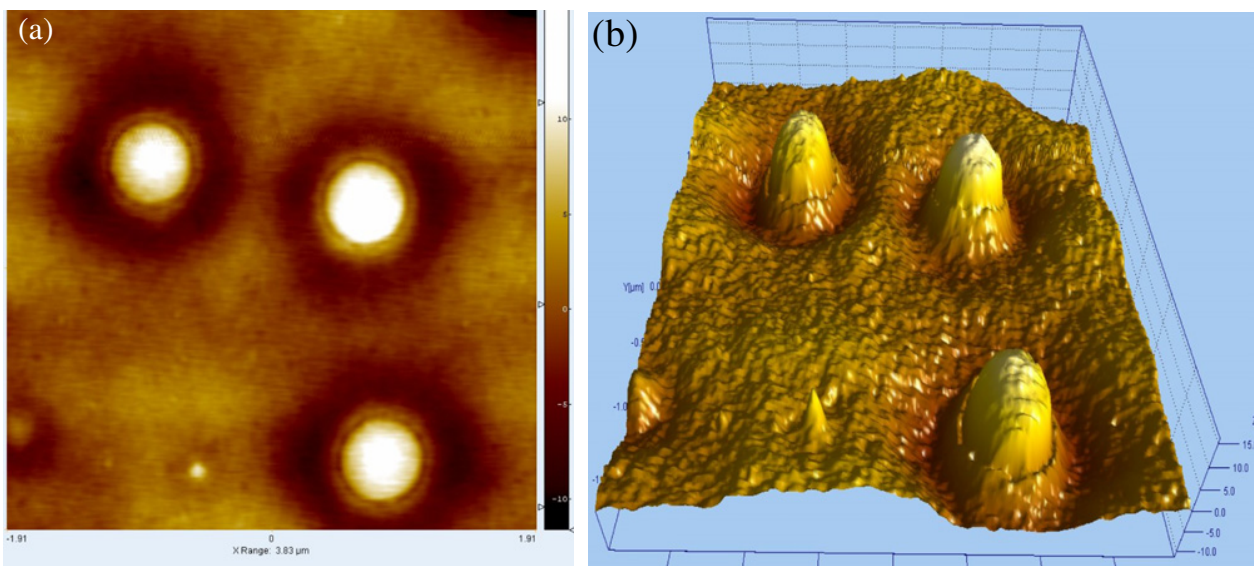

Figure 8. AFM images of bubbles under the surface 'pushing' the gelatin film upward.

These kind of features have been seen on ultrathin polystyrene films in water and they were called 'nanoindented structures' [22]. The same kind of features can also be seen in figure 7. In addition, the traces of the small bubbles that were deep inside the film (dark spots in the lower part of Figure 7(a)) appear in the 3D image as holes in a slightly raised surface, because the bubbles have left the surface.

In figure 8 it can be seen that not all the bubbles left the film. Some of them are right under the surface and provoke the raising of the surface of the gelatin, but without breaking the film.

The bubbles here have a ring structure (see the darker rings around the holes in the $2 \mathrm{D}$ image) that can be accounted for by the delay in drying of the film. The nanobubbles that diffused to the surface and exploded left behind holes as shown in figure 9 (diameter of around $300 \mathrm{~nm}$ ).

Figure 10 shows the surface of gelatin raised by a number of smaller bubbles (size $300-400 \mathrm{~nm}$ ) very close to the surface of the film, trapped in the film. No gold nanoparticles are seen in this area.

In figure 11, the traces left on the surface after the explosion of several large bubbles can be seen. The traces correspond to bubbles in the range of $2-3 \mu \mathrm{m}$ and show the very well-suited receptacles these hollow shapes may become for nanoparticles. However, in the case of non-exploded bubbles (figure 12), the surface of the film is raised by the bubbles that are close to the surface and Au nanoparticles are seen mostly outside the raised areas. When the bubbles have exploded before the film dried (figure 13) and holes were left on the surface, nanoparticles could reside separately in these spaces and pattern the surface in a quite unique way.

Figure 13(a) shows bubbles in the film as well as the traces of bubbles that left the film. As shown by the 3D image (figure 13(b)), because the bubbles were small, they have raised the gelatin surface, shaping it as tiny cones placed in the middle of the holes.

In order to elucidate the role of the experimental parameters in the formation of a particular morphology, the synthesis of the nanocomposite has been performed under various conditions. For example, by working with a high excess of chloroauric acid compared to sodium borohydride (the source of nanobubbles), the AFM images of the nanocomposite shows the presence of linear and branched gold aggregates (figure 14). This shows that, under this condition, there is a high gold-gold affinity. Nanobubbles that originate from sodium borohydrate are still present in some areas of the film as shown in figure 15. In this case, a separation of the nanobubbles and gold nanoparticles can 

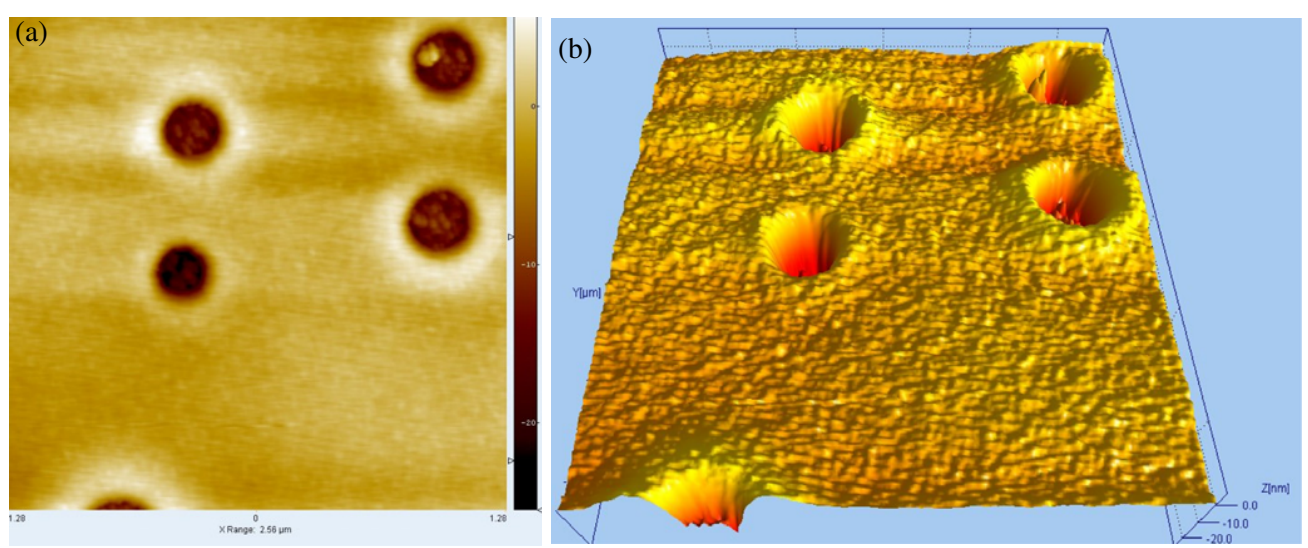

Figure 9. AFM images of the traces of nanobubbles that left the surface of the film (dark holes in the 2D image and empty cavities in the 3D image).
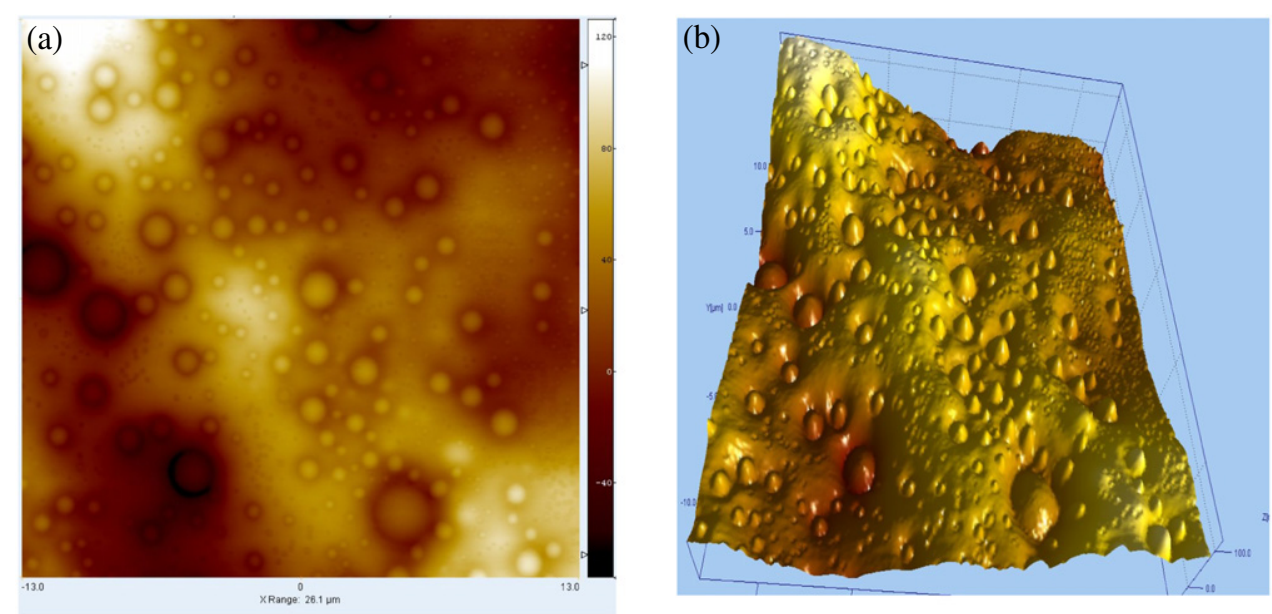

Figure 10. AFM images of small bubbles close to the surface of the film.
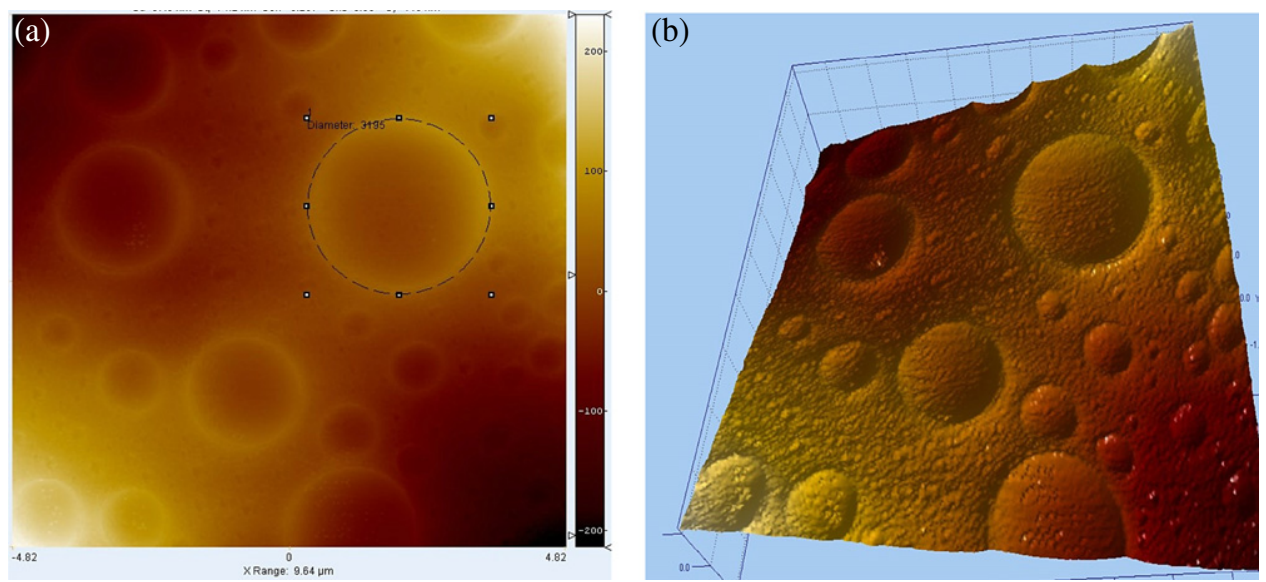

Figure 11. AFM images of the traces of large bubbles that left the surface of the film.

be seen, with no (or less) hybrid, gold-nanobubble structures. Experiments have been done under different conditions as well. Generally, the results show that, in order to have a large number of hybrid structures, a large excess of sodium borohydride has to be utilized, otherwise separate bubbles and nanoparticles will be present in the system. At this point, however, complete control of the reaction is not possible. Neither the size nor the size distribution of the bubbles can be controlled in a macro environment. In order to have a better control of the size and amount of both nanobubbles and gold nanoparticles and a more uniform morphology, the reaction has to be conducted in a more homogeneous environment and 

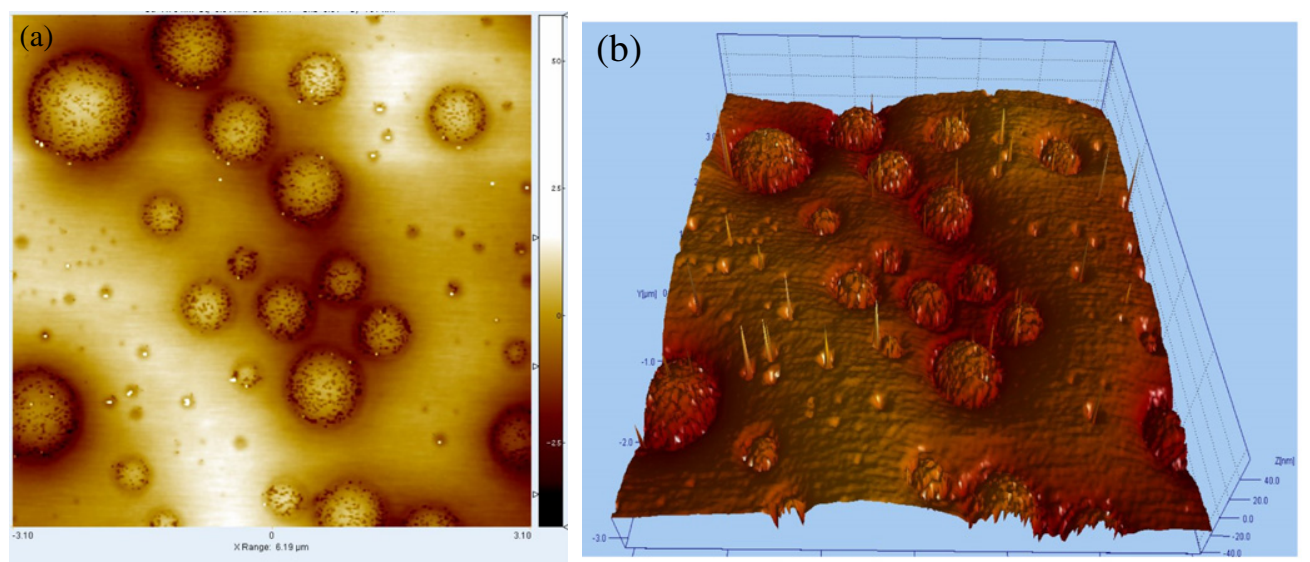

Figure 12. AFM images of Au nanoparticles around the bubbles.
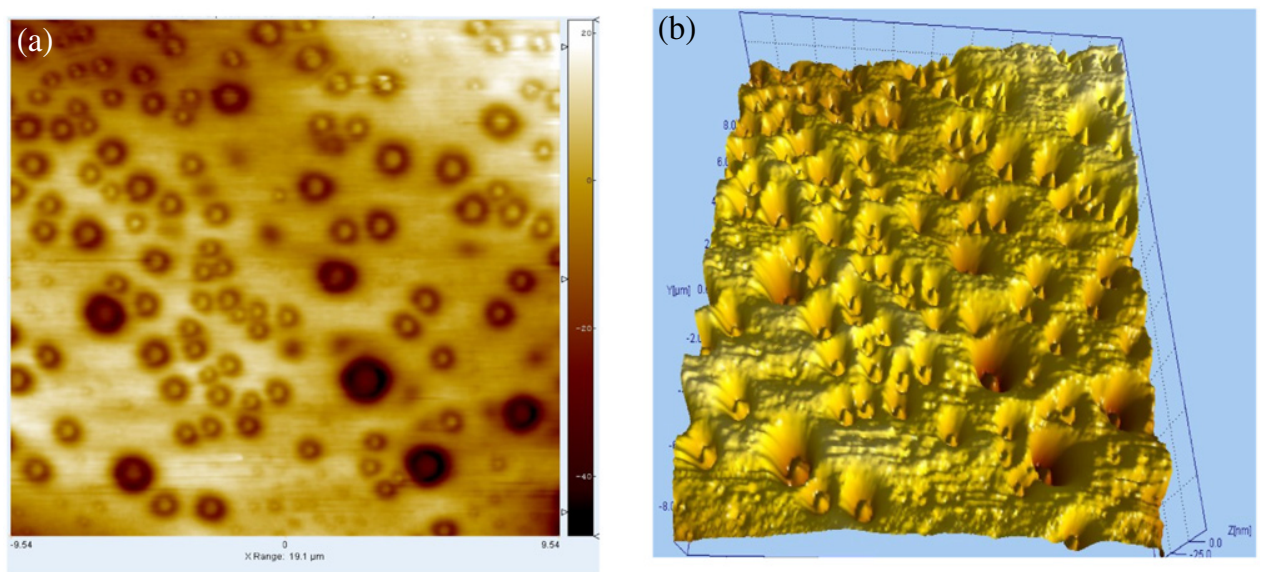

Figure 13. AFM image of small gelatin structures trapped into the holes, after the explosion of the bubbles.

a microfluidic device seems to provide a good alternative. The development of an adequate micro-reactor that will allow a good mixing of the reactants is under way.

\subsection{Simulation of the optical properties of Au-gelatin nanocomposite film with hydrogen nanobubble inclusions using discrete dipole approximation (DDA)}

Numerical simulation of the metallic nanostructures is considered the first step to design the geometrical parameters for a specific application. To achieve that goal, many computational methods based on an analytical solution of Maxwell's equations have been used to study the optical properties of the metallic structures at nanometric scale. One of them is the discrete dipole approximation (DDA) [23-26]. DDA is a computational technique for calculating the extinction and absorption efficiencies for a single particle and for assemblies of particles that are surrounded by a medium with a complex dielectric function. For particles with a size much smaller than the wavelength of the incident radiation, the quasi-static approximation is used. In this approximation, the observed surface plasmon resonance (SPR) is attributed to the excitation of the dipolar resonance. The description of the mathematical formulation of the DDA is outside the scope of this paper, but more details can be found in the references cited [23-26]. The basic idea of the DDA method is to represent the target under investigation by a three-dimensional array of $N$ dipoles which are situated on a periodic square lattice of side length $d$ which represents the inter-dipole spacing (the lattice spacing). The description of the actual volume of the target solid material depends on the number of polarizable points and the size of the square unit lattice $(d 3)$. One of the fundamental requirements in the modeling is that the inter-dipole separation should be smaller than the incident wavelength and any other structural parameters in the target under investigation. To provide an accurate description of the target's geometry, a large number of dipoles are required (typically $N>10^{4}$ ) to mimic the structural parameters of the nanostructures properly. DDA involves solving a linear equation of the form $A \cdot P=E$, where $A$ is a square matrix of the order $3 N, P$ is the induced polarization due to the interaction with the incident field and $E$ is the sum of the incident electric field and the internal field attributed to the other dipoles in the same particle. Once the polarization is obtained from the solution of the last $3 N$ complex linear equations, the optical properties can be calculated. In the calculations presented here, the open-source Fortran-90 software package (DDSCAT 7.1) was used. 


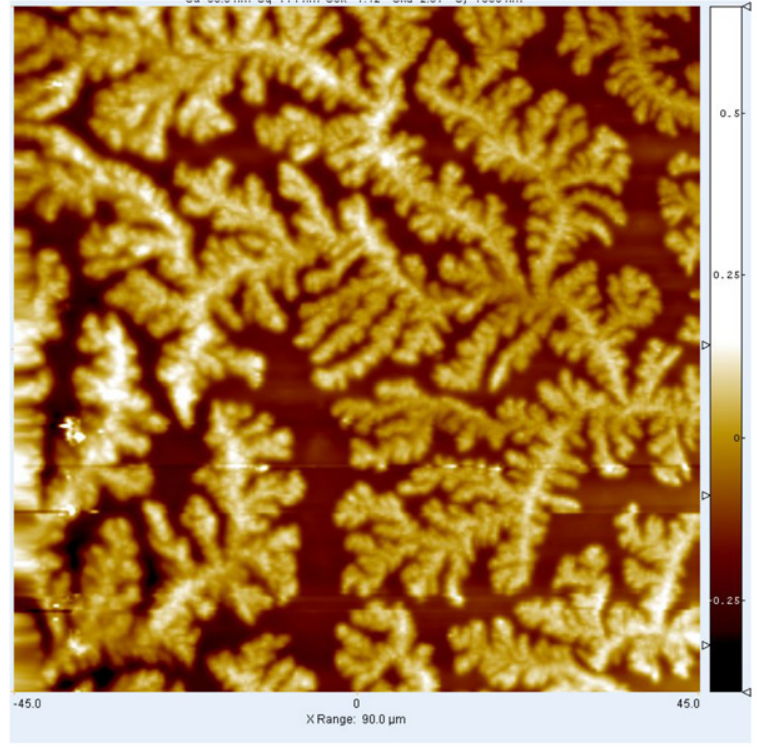

Figure 14. Linear and branched gold aggregates obtained when the reaction is conducted with an excess of chloroauric acid $\mathrm{NaBH}_{4}$ : $\mathrm{HAuCl}_{4}=2.2$.

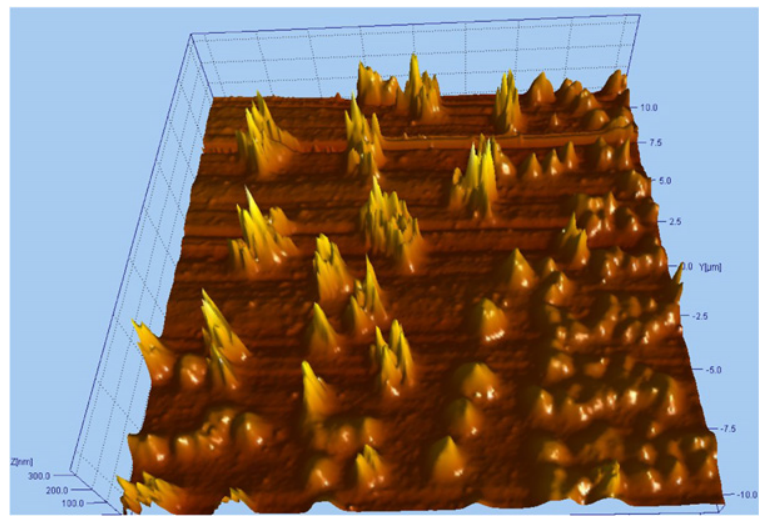

Figure 15. 3D image of the separated bubbles and gold nanoparticles when the reaction is conducted with an excess of gold precursor.

In all the simulations, the inter-dipole separation was kept constant at around $1 \mathrm{~nm}$, giving a total of 34000 dipoles per target. The chosen number of dipoles was sufficient to mimic the structure of the spherical nanoparticle well, and to achieve a proper convergence of the simulation.

Figure 16 shows the position of the Au LSPR band assuming several values for the refractive index of the environment surrounding the gold nanoparticles. The gelatin $(n=1.5)$ and the water $(n=1.33)$, when considered separately, do not correspond to the real host material. The calculated plasmonic band appears to be redshifted compared to the observed band. The hydrogen bubbles present in the real environment lower the effective refractive index and, for this reason, the best agreement with the experimental spectrum is found by assuming a hypothetical value $(n=1.2)$ which is lower than both that of gelatin and water.

By lowering the refractive index of the environment even more and assuming that the gold nanoparticles would be

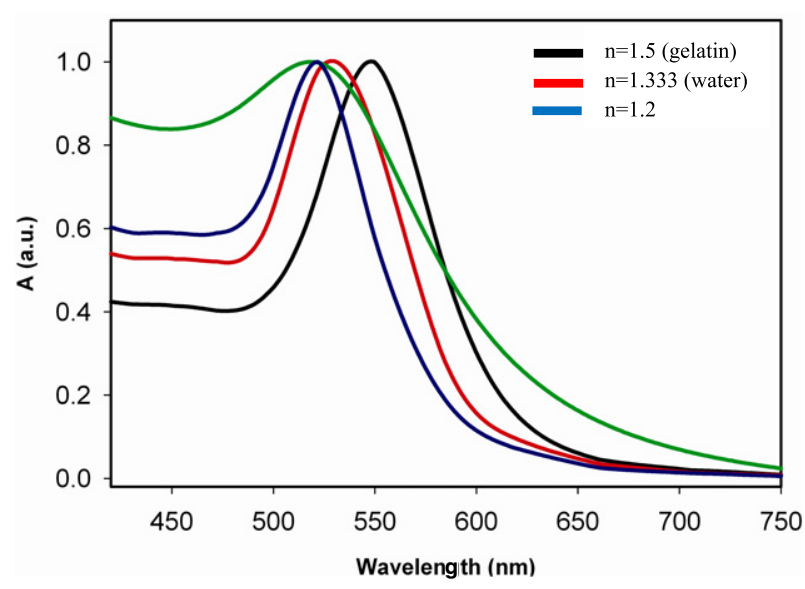

Figure 16. DDA simulation of the optical properties of gold nanoparticles of size $50 \mathrm{~nm}$ embedded in gelatin. The green curve shows the experimental band $(520 \mathrm{~nm})$ and the others show the position of the bands by assuming different refractive indices to simulate the environment.

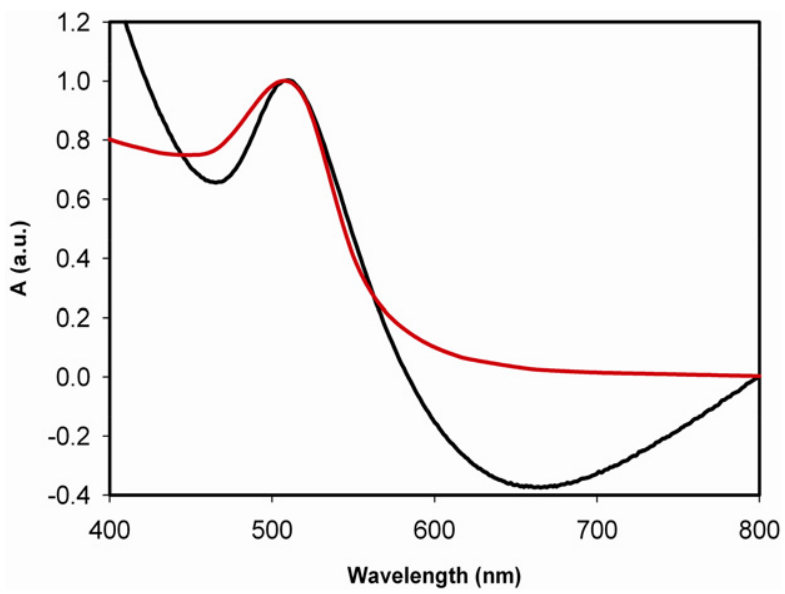

Figure 17. DDA simulation of the position of the Au LSPR band (black curve) by assuming $n=1$ (air); red curve: experimental spectrum $(510 \mathrm{~nm})$.

surrounded by air, a good agreement between the simulated and experimentalpositions of the band is found (figure 17). From this result it can be inferred that, in the nanocomposite film, there is a large amount of nanobubbles surrounding the gold nanoparticles. Indeed, the experimental value in this case corresponds to a nanocomposite prepared with a large excess of sodium borohydride, that is of hydrogen nanobubbles.

\section{Conclusion}

Gold-gelatin nanocomposites have been prepared by using sodium borohydride as a reducing agent. It is demonstrated that, under the conditions of the experiment, the nanocomposite solution as well as the film deposited on a glass substrate, includes a large amount of hydrogen micro- and nanobubbles. The presence of bubbles in the film engenders a number of interesting optical properties and morphological features that were thoroughly studied in this work and that could be useful for future applications. For example, the large traces 
left behind by the exploding bubbles are thought to become receptacles, convenient to hold the gold nanoparticles carried by the bubbles on their way to the surface of the drying film. Also, the cone-shaped gelatin features provoked by the raising bubbles may be interesting in the future for patterning applications. Application of this system for hydrogen storage, micro- and nanofluidics and drug release may also be possible. However, more work is necessary to better control the reaction in order to synthesize a nanocomposite for a specific application. It is thought that a microfluidic environment would be advantageous for this purpose.

Interestingly, DDA simulation of the optical properties points to the gaseous bubbles as being the major part of the nanoparticles' environment when a large excess of sodium borohydride is used for the synthesis. The results of this work are highly relevant to the new and exciting field of micro- and nanobubbles at surfaces and interfaces of films.

\section{References}

[1] Caseri W 2009 Inorganic nanoparticles as optically effective additives for polymers Chem. Eng. Commun. 196 549-72

[2] Srivastava S, Haridas M and Basu J K 2008 Optical properties of polymer nanocomposites Bull. Mater. Sci. 31 213-7

[3] Sanchez C, Lebeau B, Chaput F and Boilot J-P 2003 Optical properties of functional hybrid organic-inorganic nanocomposites Adv. Mater. 15 1969-94

[4] Li S, Lin M M, Toprak M S, Kim D K and Muhammed M 2010 Nanocomposites of polymer and inorganic nanoparticles for optical and magnetic applications Nano Rev. 15214

[5] Bin Ahmad M, Lim J J, Shameli k, Ibrahim N A and Tay M Y 2011 Synthesis of silver nanoparticles in chitosan, gelatin and chitosan/gelatin bionanoparticles by a chemical reducing agent and their characterization Molecules $167237-48$

[6] Darroudi M, Bin Ahmad M, Abdullah A H and Ibrahim N A 2011 Green synthesis and characterization of gelatin-based and sugar-reduced silver nanoparticles Int. J. Nanomed. 6 569-74

[7] Zhang J-J, Gu M-M, Zheng T-T and Zhu J-J 2009 Synthesis of gelatin-stabilized gold nanoparticles and assembly of carboxylic single-walled carbon nanotubes/Au composites for cytosensing and drug uptake Anal. Chem. 81 6641-8

[8] Liu Y, Liu X and Wang X 2011 Biomimetic synthesis of gelatin polypeptide-assisted noble metal nanoparticles and their interaction study Nanoscale Res. Lett. 6 22-32

[9] Cohen Simonsen A, Lyngs Hansen P and Klösgen B 2004 Nanobubbles give evidence of incomplete wetting at hydrophobic interface J. Colloid Interface Sci. 273 291-9
[10] Zhang X H, Khan A and Ducker W A 2007 A nanoscale gas state Phys. Rev. Lett. 98136101

[11] Huang C, Jiang J, Lu M, Sun L, Meletis E I and Hao Y 2009 Capturing electrochemically evolved nanobubbles by electroless deposition. A facile route to the synthesis of hollow nanoparticles Nano Lett. 9 4297-301

[12] Hui F, Li B, He P, Hu J and Fang Y 2009 Electrochemical fabrication of nanoporous polypyrrole film on HOPG using nanobubbles as templates Electrochem. Commun. $11639-42$

[13] Zhang L, Xia D and Shen Q 2006 Synthesis and characterization of $\mathrm{Ag} @ \mathrm{TiO}_{2}$ core-shell nanoparticles and $\mathrm{TiO}_{2}$ nanobubbles J. Nanoparticle Res. $823-8$

[14] Hampton M A and Nguyen A V 2010 Nanobubbles and the nanobubble bridging capillary force Coll Interface Sci. 1 1-19

[15] Seddon J R T and Lohse D 2011 Nanobubbles and micropancakes: gaseous domains on immersed substrates J. Phys.: Condens. Matter 23133001

[16] Link S and El-Sayed M A 1999 Size and temperature dependence of the Plasmon absorption of colloidal gold nanoparticles J. Phys. Chem. B 103 4212-7

[17] Philip D 2008 Synthesis and spectroscopic characterization of gold nanoparticles Spectrochim. Acta A 71 80-5

[18] Link S and El-Sayed M A 1999 Spectral properties and relaxation dynamics of surface plasmon electronic oscillations in gold and silver nanodots and nanorods J. Phys. Chem. B 103 8410-26

[19] Neupane N P, Lee S J, Park I S, Lee M H, Bae T S, Kuboki Y, Uo M and Watari F 2011 Synthesis of gelatin-capped gold nanoparticles with variable gelatin concentration J. Nanopart. Res. 13 491-8

[20] Yang H, Wang Y, Regenstein J M and Rouse D B 2007 Nanostructural characterization of catfish skin gelatin using atomic force microscopy J. Food Sci. 72 C430-40

[21] Wang Y, Yang H and Regenstein J M 2008 Characterization of fish gelatin at nanoscale using atomic force microscopy Food Biophys. 3 269-72

[22] Wang Y, Bhushan B and Zhao X 2009 Nanoindents produced by nanobubbles on ultrathin polystyrene films in water Nanotechnology 20045301

[23] Draine B T 1988 The discrete-dipole approximation and its application to interstellar graphite grains Astrophys. $J$. 333 848-72

[24] Draine B T and Flatau P J 1994 Discrete dipole approximation for scattering calculations J. Opt. Soc. Am. A 11 1491-9

[25] Draine B T and Goodman J J 1993 Beyond Clausius-Mossotti-wave propagation on a polarizable point lattice and the discrete dipole approximation Astrophys. J. $\mathbf{4 0 5}$ 685-97

[26] Draine B T and Flatau P J 1994 Discrete-dipole approximation for periodic targets: theory and tests J. Opt. Soc. Am. A 11 2693-703 